REPRODUCTIVE ETHICS

\title{
The rhythm method and embryonic death
}

\section{Bovens}

$J$ Med Ethics 2006;32:355-356. doi: 10.1136/jme.2005.013920

Some proponents of the pro-life movement argue against morning after pills, IUDs, and contraceptive pills on grounds of a concern for causing embryonic death. What has gone unnoticed, however, is that the pro-life line of argumentation can be extended to the rhythm method of contraception as well. Given certain plausible empirical assumptions, the rhythm method may well be responsible for a much higher number of embryonic deaths than some other contraceptive techniques.

Correspondence to: L Bovens, London School of Economics and Politica Science, Department of Philosophy, Logic, and Scientific Method, Houghton Street, London WC2A 2AE, UK; L.

Bovens@|se.ac.uk

Received 9 August 2005 Accepted for publication 11 August 2005 t has not gone unnoticed by advocates of the pro-life movement that if one is concerned about abortion because of the moral turpitude of killing embryos (and fetuses) then one should also be concerned about various contraceptive techniques. Certainly, they say, one should be concerned about the morning after pill and intrauterine devices (IUDs), since these techniques block the implantation of a conceived ovum. This argument has been extended to the contraceptive pill as well. The contraceptive pill (i) changes the cervical mucus so that the passage of the sperm is blocked, (ii) inhibits ovulation, and (iii) affects the endometrium so that the uterus is not a hospitable environment for implantation. Of course this third route is only operational in preventing pregnancy if the first and second routes fail. It is not known in what percentage of cases the pill fails to block the sperm and fails to inhibit ovulation and is effective only because it manages to block implantation. It is argued, however, that even if this is rarely the case, a great number of embryonic deaths are caused due to this aspect of pill usage. Randy Alcorn calculates that "even an infinitesimally low portion ( say one hundredth of one per cent) of 780 million pill cycles per year globally could represent tens of thousands of unborn children lost to this form of chemical abortion annually". ${ }^{\prime \prime}$

A concern for consistency has pushed advocates of the pro-life position into opposing all contraceptive techniques that cause embryonic deaths. Catholics might welcome this, since the official position of the church is that, aside from the rhythm method, no contraceptive techniques are permissible. This benefit is questionable. What has gone unnoticed is that, if one is willing to make a few relatively innocent assumptions, then the rhythm method may well be responsible for massive embryonic death and the same logic that turned pro-lifers away from morning after pills, IUDs and pill usage, should also make them nervous about the rhythm method.

The first assumption is that there are a great number of conceptions that never result in missed menses. There are estimates that only $50 \%$ of conceptions actually lead to pregnancies. The second assumption is that, even in clinical trials, the rhythm method can fail due to the fact that a pregnancy results from sexual intercourse on the last days before and the first days after the prescribed abstinence period. Estimates of the effectiveness of the rhythm method vary in the literature, but let us set its effectiveness for clinical trials at $90 \%$-that is, conscientious rhythm method users can expect one pregnancy in ten woman years. The third assumption is that there is a greater chance that a conception will lead to a viable embryo if it occurs in the centre interval of the fertile period than if it occurs on the tail ends of the fertile period. This assumption is not backed up by empirical evidence, but does have a certain plausibility. From assumption one, we know that there is a high embryonic death rate. It seems reasonable to assume that an embryo that results from an "old" ovum (that is waiting at the end of the fertile period) or an "old" sperm (that is still lingering on from before ovulation), and that is trying to implant in a uterine wall that is not at its peak of receptivity, is less viable than an embryo that comes about in the centre interval of the fertile period. Let us make a conservative guess that the chance that an embryo conceived in the centre interval of the fertile period, which coincides with the abstinence period in the rhythm method-let us call this "the heightened fertility (HF) period"-is twice as likely to be viable as an embryo conceived at the tail ends of the fertile period.

So now let us run the argument. We know that even conscientious rhythm method users get pregnant. Conception may occur due to intercourse during the tail ends of the fertile period and the conceived ovum may turn out to be viable. Rhythm method users try to avoid pregnancy by aiming at the period in which conception is less likely to occur and in which viability is lower. So their success rate is due not only to the fact that they manage to avoid conception, but also to the fact that conceived ova have reduced survival chances. Just like in the earlier case of pill usage, we do not know in what percentage of cases the success of the rhythm method is due to the strictly contraceptive workings of the technique and in what percentage of cases it is due to the reduced survival chances for the conceived ovum. None the less, along with Alcorn, one could argue that even if the latter mechanism has only limited effectiveness, it remains the case that millions of rhythm method cycles per year globally depend for their success on massive embryonic death.

Abbreviation: IUD, intrauterine device 
Let us try to make the argument more vivid. Pro-lifers oppose IUDs because their main mode of operation is to make embryonic death likely. Now suppose that we were to learn that the success of the rhythm method is actually due, not to the fact that conception does not happen-sperm and ova are much more long lived than we previously thought-but rather because the viability of conceived ova outside the HF period is minimal due to the limited resilience of the embryo and the limited receptivity of the uterine wall. If this were the case, then one should oppose the rhythm method for the same reasons as one opposes IUDs. If it is callous to use a technique that makes embryonic death likely by making the uterine wall inhospitable to implantation, then clearly it is callous to use a technique that makes embryonic death likely by organising one's sex life so that conceived ova lack resilience and will face a uterine wall that is inhospitable to implantation. Furthermore, if one is opposed to IUDs because their main mode of operation is to secure embryonic death, then, on the assumption that one of the modes of operation of the pill is to make embryonic death likely, one should be equally opposed to pill usage. This is essentially Alcorn's argument and assuming that the empirical details hold, consistency does indeed drive IUD opponents in this direction. If, however, our empirical assumptions about the rhythm method hold, then one of its modes of operation is also that it makes embryonic death likely. And if embryos are unborn children, is it not callous indeed to organise one's sex life on the basis of a technique whose success is partly dependent on the fact that unborn children will starve because they are brought to life in a hostile environment?

What is the expectation of embryonic death for rhythm method users? Our first assumption was that only half of the embryos are viable. I take it that this value holds for populations using no contraception and not distinguishing between HF and non-HF periods (or using contraceptive techniques that do not distinguish between HF and non-HF periods). What is not known is what proportion of embryos are conceived during the HF period as opposed to outside the HF period. Since it is reasonable to assume that only a minority of embryos are conceived outside the HF period, let us make a broad estimate that between $1 / 10$ and $1 / 3$ are so conceived. Then, by our third assumption - that is, that the chance of the viability is twice as high for an embryo conceived during the HF period as for an embryo conceived outside of the HF period, we can calculate that the chance of viability outside the HF period ranges roughly from one in four to one in three. ${ }^{i}$ So, on average, for every pregnancy that results from a conception outside the HF period, there are two to three embryonic deaths. And hence, by our second assumption - that is, that rhythm users may expect one pregnancy in ten woman years, it follows that we can expect two to three embryonic deaths in ten woman years. If all of Alcorn's 780 million pill users were to switch to the rhythm method, then these converts would be causing, in his own words, the deaths not of tens of thousands, but of millions of unborn children.

So what is the alternative? If one is concerned about minimising embryonic death, then one should avoid types of contraception whereby each unintended pregnancy (due to its failure) comes at the expense of a high embryonic death rate. ${ }^{\text {ii }}$ Given our first assumption, a condom user (who makes no distinction between HF and non-HF periods) can count on one embryonic death for each unintended pregnancy. A rhythm

'By the probability calculus, the probability of viability $(p)$ equals the conditional probability of viability given that the conception occurred outside the HF period (q) times the probability that the conception occurred outside the HF period $(r)$ plus the conditional probability of viability given that the conception occurred during the HF period $(2 q)$ times the probability that the conception occurred during the HF period $(1-r)$. Hence, $p=q r+2 q(1-r)$. We set $p=1 / 2$ by assumption one and let $r \in[1 / 10,1 / 3]$. Hence $q \in[10 / 38,3 / 10] \approx[1 / 4,1 / 3]$. method user, however, should count on two to three embryonic deaths for each unintended pregnancy. Assuming a success rate of $95 \%$ for condom usage, we can count on an expectation of .5 pregnancies in 10 years. Hence, the expectation of embryonic death is .5 per ten years for a condom user, which is substantially lower than the expectation of two to three embryonic deaths per ten years on the rhythm method. Even a policy of practising condom usage and having an abortion in case of failure would cause less embryonic deaths than the rhythm method.

So how can this argument be blocked? First, one could say that the empirical data are questionable. However, the result really depends on the simple assumption that embryos conceived outside the HF period are less viable than embryos conceived during the HF period. If this is the case, then the success of the rhythm method is contingent on a higher embryonic death rate and so every pregnancy due to a failure of the technique will come at the expense of a higher embryonic death rate - and this is all that is needed to get the argument off the ground. Second, one could be concerned about the death of an embryo due to an abortion but not due to IUD usage, because not providing the right environment for embryonic growth is less of a direct action than performing an abortion. This would bring in the intricacies of the action/omission doctrine. I am dubious that enough can be gleaned from the action/omission doctrine to support this distinction, but this is not the place to turn to this discussion. Third, one might draw a moral distinction between techniques that cause embryonic death (such as abortion and IUDs) and techniques that employ a mixed approach of preventing conception and increasing the likelihood of embryonic death in case conception occurs (such as the contraceptive pill and the rhythm method). There may indeed be a psychological distinction, similar to the comfort a person in a firing squad receives from not knowing that it was his bullet that killed the victim, but I do not think that this distinction has any normative force. Fourth, one might try to make a distinction between causing an inhospitable environment for embryonic survival (as in IUD and pill usage) and restricting the possibility of conception to a time when the environment is inhospitable for embryonic survival (as in the rhythm method). Again, the former may be considered to be more of a direct action than the latter, but once again, I think that this would be asking more from the action/omission doctrine than it can deliver.

And finally, one person's modus ponens is another person's modus tollens. One could simply conceive of this whole argument as a reductio ad absurdum of the cornerstone of the argument of the pro-life movement, namely that deaths of early embryos are a matter of grave concern.

\section{ACKNOWLEDGEMENT}

I am grateful for comments by John Harris and Leah McClimans, and Michael Otsuka. My research was supported by the Alexander von Humboldt Foundation, the Federal Ministry of Education and Research, and the Program for the Investment in the Future (ZIP) of the German Government through a Sofja Kovalevskaja Award.

\section{REFERENCES}

1 Alcorn R. Does the birth control pill cause abortions? http://www.epm.org/ articles/bcp5400.html (accessed 14 Sept 2005)

2 Harris J. Stem cells, sex and procreation. Camb Q Healthc Ethics $2003 ; 12: 335-72$.

iijohn Harris makes a similar argument for procreation. ${ }^{2}$ If one is genuinely concerned about embryonic death, then one should choose reproductive techniques that minimise embryonic death. If IVF had developed to the point that a pregnancy could be brought about at minimal cost of embryonic death, then one would be required to refrain from reproducing through sexual intercourse, because it would come at a needlessly high cost of embryonic death. Harris (Harris, ${ }^{2}$ p 346) takes this to be a reductio ad absurdum of the reverence with which the pro-life movement treats embryos in the discussion about stem cell research. 